\title{
VARIOUS METHODS OF MANUFACTURING SUPERCONDUCTING ACCELERATING CAVITIES
}

\author{
C. Benvenuti, Ph. Bernard, D. Bloess, E. Chiaveri, \\ C. Hauviller and W. Weingarten \\ CERN, European Organization for Nuclear Research \\ 1211 Geneva 23, Switzerland
}

\begin{abstract}
We report on experience in superconducting cavity production methods gained in shaping, joining and thin film coating with various materials and techniques $(\mathrm{Pb}, \mathrm{Nb}$, $\mathrm{Nb}_{3} \mathrm{Sn}, \mathrm{NbN}, \mathrm{NbTiN}$ ) with emphasis on their potential to reduce mass production costs.
\end{abstract}

\section{INTRODUCTION}

Production technology of superconducting (sc) cavities has entered a new phase: the number of manufactured cavities has jumped from a low value (recyclotrons, heavy ion accelerator facilities, intermediate sc RF systems such as in TRISTAN, HERA) to a large series of hundreds (CEBAF, LEP2) and even thousands (proposed TESLA). This necessitates reconsideration of production and quality control methods with respect to performance requirements and cost effectiveness.

The maximum attainable gradient is not necessarily that for which the construction cost is a minimum. If the total RF voltage, $V$, and the current are fixed, the total investment costs are given by three terms. The first one is proportional to the total length $L$ of the cavities and cryostats ("linear" costs), the second one is proportional to the total RF power $P_{\mathrm{RF}}$ to be transmitted to the beam (independent of $L$ ), and the third one is proportional to the cooling power ("cryogenic" costs), which is essentially the power $P_{\mathrm{d}}$ dissipated in the RF cavities. Since $V=E_{\mathrm{a}} \cdot L$ is constant, $E_{\mathrm{a}}$ being the accelerating gradient, the first term is inversely proportional to $E_{\mathrm{a}}$. If $E_{\mathrm{a}}$ is increased, the RF losses will go up quadratically with $E_{\text {a }}$. On the other hand the length $L$ is inversely proportional with $E_{\mathrm{a}}$, because $V$ is constant. Hence $P_{\mathrm{d}}$ increases only linearly with $E_{\mathrm{a}}$ and consequently the cooling power to be installed. The cost minimum is located where the first and third term are equal. The following example is based on numbers, which are close to CERN's experience $^{1}$ for LEP. Fig. 1 displays these three terms and their dependence on $E_{\mathrm{a}}$. It shows that a gradient between 8 and $10 \mathrm{MV} / \mathrm{m}$ is an optimum choice for this particular application.

However, having in mind even larger applications, such as the sc linear collider TESLA under design, at higher frequency $(1.3 \mathrm{GHz})$ and higher energy $(500 \mathrm{GeV})$, costs must be reduced substantially. The cooling power may be reduced (technical Q-values being in the $10^{9}$ range) by slowly pulsing the accelerator, which reduces the cryogenic costs. One can see from Fig. 1 that for a duty cycle of $1 \%$ (close to that envisaged in TESLA) a gradient of $25 \mathrm{MV} / \mathrm{m}$ would yield the cost minimum provided that the linear 
costs go down by a factor of 16 . To obtain such a factor would be a challenge. Under these assumptions the total costs (for $V=2 \mathrm{GV}$ ) would go down by a factor of 40 .

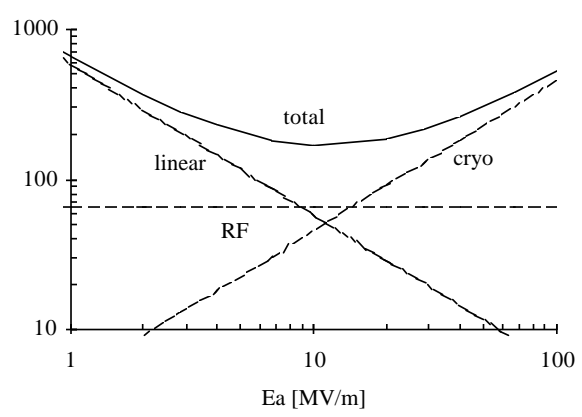

Figure 1. Estimate of investment costs for an sc RF system $(f=352 \mathrm{MHz}, V=2 \mathrm{GV}, 12 \mathrm{MW}$ RF power, 5 $\mathrm{W} / \mathrm{m}$ standby heat load, $\left.Q_{0}=4 \cdot 10^{9}\right)$ : The total costs are the sum of three terms, linear, RF and cryogenic costs.

The purpose of this paper is to review the state of the art of producing sc cavities. It will focus on the manufacturing of the sc accelerating structure itself. Methods with the potential to save costs will be looked at more closely.

\section{THE MECHANICAL STRUCTURE}

The sc cavity consists of two essentials: the "mechanical structure" and the layer which carries the RF current, the "sc surface".

Table 1. Comparison of niobium and copper 1)

\begin{tabular}{|c|c|c|}
\hline & Cavity grade $\mathrm{Nb}$ & OFE Cu \\
\hline Thermal conductivity@4.2 K [W/(mK)] & $10(\mathrm{RRR}=40)-100(\mathrm{RRR}=400)$ & $400-500$ \\
\hline Electrical resistivity @295 K [10-8 1/2m] & 14.4 & 1.68 \\
\hline Density $\left[\mathrm{g} / \mathrm{cm}^{3}\right]$ & 8.57 & 8.96 \\
\hline Linear expansion $\left(\mathrm{L}_{273}-\mathrm{L}_{4.2}\right) / \mathrm{L}_{4.2}[\% o]$ & 1.43 & 3.3 \\
\hline Atomic weight & 92.91 & 63.54 \\
\hline Atomic number & 41 & 29 \\
\hline Melting point [_C] & $2468-2497$ & 1080 \\
\hline Recrystallization temperature [_C] & $830-1230$ & $400-600$ \\
\hline Stress relieving temperature [_C] & $780(1 \mathrm{~h})$ & $200-250$ \\
\hline Tensile strength $\left[\mathrm{N} / \mathrm{mm}^{2}\right]$ & $207-274$ & $196-245^{3)}$ \\
\hline Elongation [\%] & $6-42$ & $6-50$ \\
\hline Yield point $\left[\mathrm{N} / \mathrm{mm}^{2}\right]$ & $20^{2)}-196$ & $15-300$ \\
\hline 1) Some data are taken from refs. 2,3 & $10^{-8}$ Torr & \\
\hline
\end{tabular}

The mechanical structure has to provide three features. At first it must be sufficiently stable and rigid at low temperatures to sustain with a safety margin the pressure in the helium tank (usually 1 bar) outside, the radiation pressure inside and to block mechanical vibrations. Secondly the thermal conductivity must be such that heat is effectively removed without generating too large temperature gradients (thermal stability). Thirdly it must be shaped economically with reasonable effort and joints between pieces must be vacuum tight and reliable.

Up till now two materials have been used for the mechanical structure; niobium and copper (Table 1). Niobium can simultaneously provide the mechanical structure and the sc surface. In addition it is the element with the largest transition temperature $T_{\mathrm{c}}$. Copper is relatively cheap, can be manufactured and shaped without tremendous effort and has a large thermal conductivity. 


\section{Mechanical Stability and Rigidity}

The wall thickness of the mechanical structure assures its mechanical stability. It has to be designed according to contradicting constraints. Thin walls are less costly, easier to handle and effectively cooled, but on the other hand prone to buckling from pressure spikes inside the He tank and mechanical and ponderomotive oscillations (cf. below). Modern accelerating structures are made from several $\mathrm{mm}$ thick material (niobium or copper). A Finite Element Analysis software such as CASTEM ${ }^{4}$ allows the mechanical behaviour of the structure, in particular the buckling modes, the maximum tolerable pressure, and the natural resonant frequencies to be understood (Fig. 2) 5 . Main modes of vibration can be suppressed by imposing forces of constraint on well-chosen points of maximum amplitude. A complete suppression of all the mechanical vibration modes is impossible. These modes add phase shifts on the tuner feedback loop and can make it unstable. A way out is to shift their resonant frequencies to larger values beyond the tuner bandwidth. This could be done by increasing the rigidity of the stiffening bars and hence the resonant frequency.

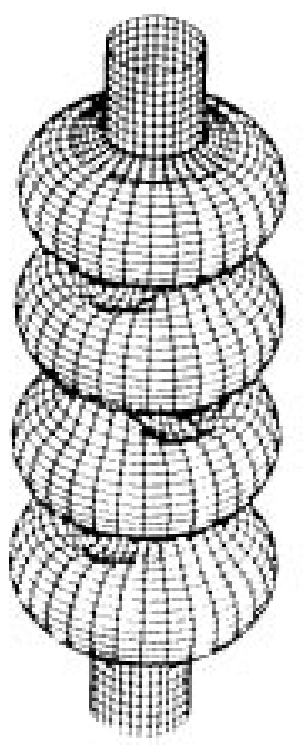

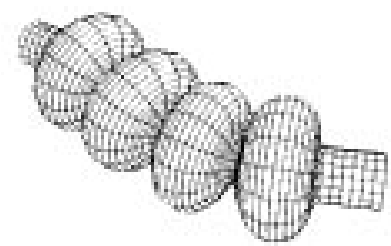

MODE 2
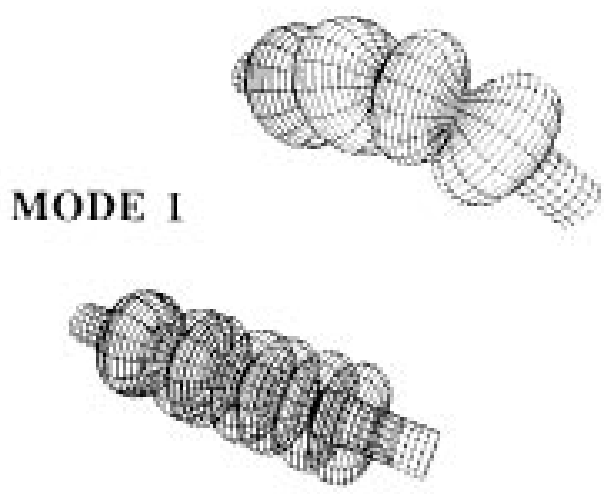

Figure 2. CASTEM Output: left: Mode of buckling for four cell $1.5 \mathrm{GHz}$ copper cavity with $2 \mathrm{~mm}$ wall thickness (critical pressure is 23.6 bar with elastic limit of copper of $100 \mathrm{~N} / \mathrm{mm}^{2}$ ); right: mechanical resonances at 146.2 $\mathrm{Hz}$ (transversal with two nodes, mode 1) and $225.3 \mathrm{~Hz}$ (longitudinal, mode 2).

Ponderomotive forces 6 depend on the square of the accelerating gradient. By increasing the accelerating gradient, the cavity is driven out of resonance, for example by the radiation pressure acting on the cavity wall (Lorentz force). It takes a certain time before the wall moves (there is a delay). The RF amplitude will fall, and the resonant frequency will be shifted back to tune again, which closes the loop. The delays present can make the system unstable.

\section{Thermal Conductivity}

The temperature $T$ near a localized heat source falls inversely proportional to the thermal conductivity $\lambda$ and the distance $r$ from that heat source. This may be a normalconducting inclusion, a poorly-cooled welding bead, peeled-off flake etc., or a region of electron impact. If the source dissipates too much heat, the transition temperature of the sc surface can be surpassed, by which effect the stored energy (100 J for a LEP cavity at $6 \mathrm{MV} / \mathrm{m}$ accelerating gradient) is dumped near the defect (quench). The cavity can only be operated at a gradient well below the quench. Therefore the thermal conductivity near $T_{\mathrm{c}}$ of the material in the intimate vicinity of the heat source is the important parameter.

It becomes immediately clear that, to increase the maximum gradient, the number and size of the surface defects have to be reduced, and, on the other hand, sc metal of increased thermal conductivity must be made available (thermal stabilization of defects). 
It is known 7,8 that the thermal conductivity of niobium is most severely affected by interstitial impurities such as oxygen, nitrogen and carbon. These impurities can be considerably reduced by repeated furnace treatments on the manufacturer's premises ${ }^{9-11}$ and/or solid-state gettering by wrapping a foil of metal with a large affinity to these impurities around the cavity in a furnace at 1200-1400_C. Yttrium and titanium are used as getter material ${ }^{12,13}$.

\section{Shaping and Joining Techniques}

There exists a wealth of knowledge on shaping and joining techniques $2,14-25$, which will not be repeated here. Niobium can be machined by the usual high speed steel tools. It turns very much like lead or soft copper. It has nevertheless the tendency to gall; tool angles, cutting speed and lubrication have to be carefully chosen. Normal techniques of metal spinning are applied, and the metal takes up the contour of the former. Deep drawing is also used and gives satisfactory results (no "orange peel") for fine grain material (ASTM < 4).

Hydroforming is a common manufacturing procedure (e.g. production of bellows). The principle is to push the part against a rigid die by applying a large pressure through a liquid. Monolithic pieces can be produced, and welding can be avoided. Reproducibility is superior compared to welded parts, but the tooling is expensive. This fact is of no consequence for a large series. However, repeated annealing is necessary and increases the cost. Cavities in the frequency range from $300 \mathrm{MHz}$ to $3 \mathrm{GHz}$ have been produced from OFE (Oxygen Free Electronic grade) copper tubes 22 . The frequency of the fundamental mode of $1.5 \mathrm{GHz}$ five-cell cavities has a standard deviation of $\pm 0.3 \% o^{26}$. The final deformation is more than $200 \%$. The ultimate elongation of copper being only of the order of $50 \%$ (Table 1), this process has been achieved in several stages: swaging has been followed by three expansion and intermediate annealing steps at $600{ }^{\circ} \mathrm{C}$ for one hour.

Swaging means that an oil-pressurized membrane pushes the annealed copper tube on to the internal core creating a toroidal groove. The problem is to prevent plastic buckling due to high compressive stresses creating ripples. The process has been modelled by the BOSOR $5^{27}$ software and experimentally checked. Good correlation has been obtained for the critical buckling values in the case of uniform thickness. Thirty-five percent reduction of the iris diameter has been achieved in only one stage with a pressure up to 650 bars.

The expansion is obtained by a multi-part die which is initially open and closed progressively during expansion. The closed die has the exact external shape of the final cavity. The internal hydraulic pressure progressively increases up to 200 bar. The number of expansion steps depends on the radial deformation needed and on the behaviour of the annealed copper. CASTEM software ${ }^{4}$ was used for modelling. A precise prediction of the behaviour during all stages has been possible.

OFE copper has high purity and high conductivity at cryogenic temperatures. The parameters of the heat treatment were determined using standard tensile tests. The heat treatment starts to influence material properties from $400{ }^{\circ} \mathrm{C}$. The hydroforming phases were then simulated on test samples (initial annealing, 32\% elongation and second annealing, 32\% elongation and third annealing).

Hydroforming niobium was first used at HEPL (Stanford) ${ }^{28}$. This metal has very similar properties to copper (Table 1) except the characteristic temperatures such as, for example, the melting point. Heat treatment should be done at $1000^{\circ} \mathrm{C}$ for about one hour. The procurement of high quality niobium tubes is not easy. Seamless tubes and rotary swaged longitudinally welded ones are presently being investigated.

Spinning is another method for cheap mass production. A seamless nine-cell 1.5 $\mathrm{GHz}$ cavity starting from a planar disk of Al has been spun without annealing 29 , and $\mathrm{Cu}$ and $\mathrm{Nb}$ nine-cell cavities are under preparation. The thickness uniformity was about a factor of two.

Galvanoplastic shaping 30 is another technique to build the whole structure without welds in one stride: an organic glass mandrel (shaped like the inner surface of the cavity) is sputter coated with a copper layer, on to which the bulk copper is deposited by the galvanoplastic technique. Subsequently the mandrel is dissolved. A $3 \mathrm{GHz}$ nine-cell cavity 
has been produced and sputter-coated with a niobium film inside. RF results are not yet published.

\section{THE SUPERCONDUCTING SURFACE}

The superconducting surface has to comply with the following three features: it must tolerate high electric fields and currents at sufficiently low RF losses (large Q-values); it must be operated at a temperature which can be produced by the evaporation of a cryogenic liquid with reasonable effort; it must be operated at a temperature sufficiently below the critical temperature $T_{\mathrm{c}}$ of the sc material (safety margin). There are three different ways of complying simultaneously with these demands, which reflect the historical progress of the technology. The most straightforward way is to use the structure material as the sc surface as well. The second one is to modify the structure material (towards larger $T_{\mathrm{c}}$ and Q-value which means less effort for cooling). The third one separates the function of the structure and the sc surface completely and opens up an avenue towards new materials for the sc surface (thin films).

\section{Identical Superconducting Surface and Structure Material}

The metal (exclusively niobium) has been machined as, for example, for the CERNKarlsruhe RF separator ${ }^{25}$, or has been shaped by deep drawing and machined, as for the Stanford HEPL recyclotron ${ }^{28}$, or is shaped by deep drawing or spun from sheet metal like, for example, the CEBAF, CERN-SPS-LEP (first phase), Darmstadt-Wuppertal, DESYHERA, KEK-TRISTAN structures. Some effort with TIG welding has been made, but EB welding of the half cups ${ }^{23}$ (preferably from the inside ${ }^{24}$ ) and irises gave more reliable results. The best results in Q-value and maximum gradient $E_{\mathrm{a}}$, obtained in an industrial series production, are shown in Fig. 3 (a) and (c).
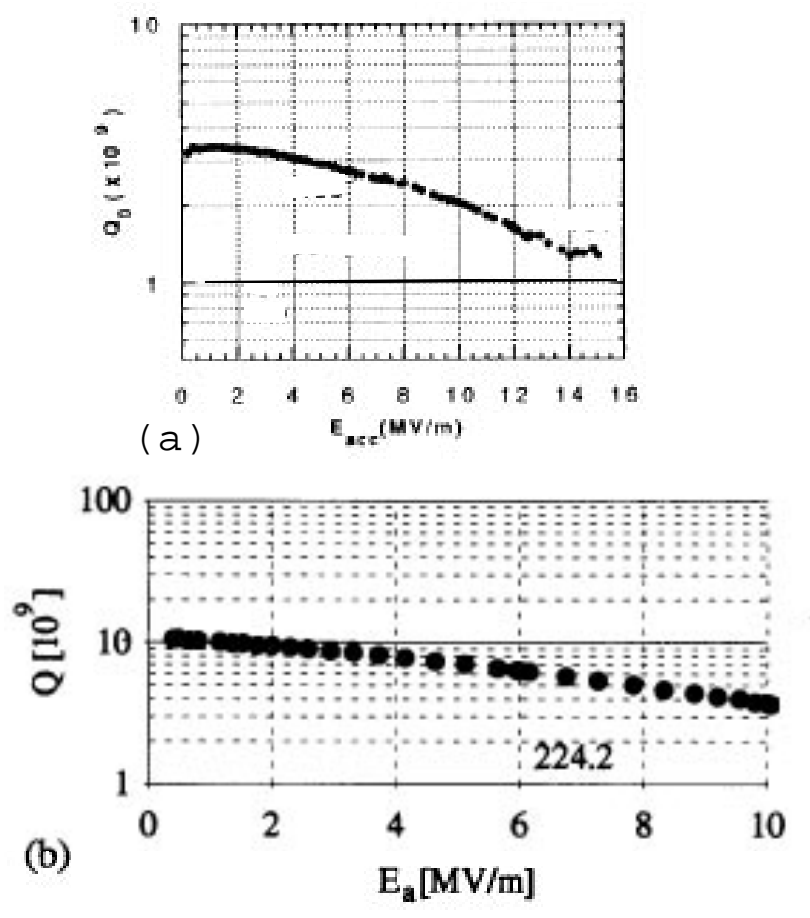

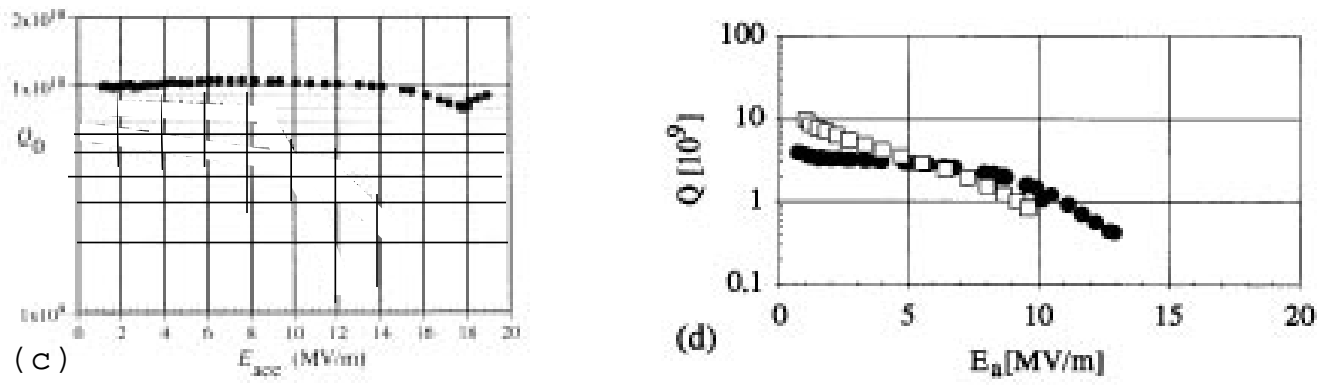

Figure 3. $\mathrm{Q}_{0}-\mathrm{E}_{\mathrm{a}}$ plots in vertical tests (best results) for cavities from serial production in industry [except (d)]: (a) a $508 \mathrm{MHz}$ five-cell niobium sheet cavity for TRISTAN ${ }^{31}$ (4.2 K); (b) a 352 four-cell niobium coated copper cavity for $\mathrm{LEP}^{32}(4.2 \mathrm{~K})$; (c) a $1500 \mathrm{MHz}$ niobium sheet cavity for CEBAF 33 (1.8 K); (d) $1500 \mathrm{MHz}$ hydroformed niobium coated copper cavity (from industrial production feasibility study) ${ }^{21}$ $(1.8 \mathrm{~K})$.

The processing of the surface. When the structure and the sc surface are identical, the properties of the sc surface pay tribute to those of the mechanical structure (inclusions from rolling and shaping, thermal conductivity). Nevertheless, the methods of surface processing have been refined in such a way that the maximum gradients obtained in multicell accelerating structures have been pushed up to $16 \mathrm{MV} / \mathrm{m}$ in a five-cell $508 \mathrm{MHz}$ cavity at $\mathrm{KEK}^{31}$ and about $22 \mathrm{MV} / \mathrm{m}$ in a nine-cell $1.5 \mathrm{GHz}$ cavity in the TESLA collaboration ${ }^{34}$. The first result wasobtained by electropolishing, heat treatment $(1.5 \mathrm{~h}$ at 700_C) to remove the embedded hydrogen, and rinsing with ultra-pure water under ultrasonic agitation. The second one was obtained by chemical polishing, solid state getter furnace treatment (to improve the thermal conductivity) before welding, rinsing with ultrapure water (under low and high pressure), and pulsed operation under high RF power (high power pulsed processing) 35 .

In the presence of hydrogen (accumulated by slow chemical polishing), cavity grade $\mathrm{Nb}$ with high thermal conductivity forms compounds which during slow cool down precipitate as hydrides at low temperatures $(\sim 100 \mathrm{~K})$ and degrade the Q-value ${ }^{36}$. These cavities have to be annealed to chase the hydrogen or the cool down has to be done fast.

Cavities with identical mechanical structure and sc surface could be taken out one by one, coated by compounds based on the structure metal with improved surface properties (as $\mathrm{Nb}_{3} \mathrm{Sn}$, see below), and retrofitted into the accelerator again.

Acceptance rates in initial vertical tests in an industrial series production for CEBAF are $98 \%\left(E_{\mathrm{a}}=5 \mathrm{MV} / \mathrm{m} \text { and } Q=2.4 \cdot 10^{9}\right)^{33}$.

\section{Superconducting Surface from Reprocessed Structure Material}

A15 and B1 compounds. The surface resistance $R_{\mathrm{S}}$ is composed of the theoretically well understood BCS surface resistance $R_{\mathrm{BCS}}$ (which can be calculated by using the BCS theory) and the residual surface resistance $R_{\text {res. }}$. At a given operating temperature $R_{\mathrm{BCS}}$ falls exponentially with increasing Cooper pair energy $2 \Delta$ (2 $2 \Delta$ is the energy gap of the sc metal). The BCS theory tells us that $\Delta$ is proportional to $T_{\mathrm{c}}, \Delta=3.5 \mathrm{k} T_{\mathrm{c}}$, hence superconductors with a larger pairing energy $\Delta$ should allow a smaller surface resistance at the same temperature, provided the pre-exponential constant is about the same. This is the reason why both the $\mathrm{B} 1$ and $\mathrm{A} 15$ compounds $\mathrm{NbN}$ and $\mathrm{Nb}_{3} \mathrm{Sn}$ are also being investigated for RF applications.

Table 2. BCS surface resistance of some metals

\begin{tabular}{|c|c|c|c|c|}
\hline & $T \mathrm{c}[\mathrm{K}]$ & $A$ & $n$ & $\Delta^{\prime}$ \\
\hline $\mathrm{Nb}(R R R=100)$ & 9.25 & 105 & 2 & 18 \\
\hline $\mathrm{Nb}_{3} \mathrm{Sn}$ & 18.1 & 105 & 2 & 40 \\
\hline $\mathrm{Pb}$ (Ref. 37, Fig. 1) & 7.2 & 74 & 1.75 & 14.6 \\
\hline $\mathrm{PbSn}(4 \text { at } \% \mathrm{Sn})^{38}$ & 7.5 & 68.5 & 1.9 & 15.1 \\
\hline
\end{tabular}


The BCS surface resistance (in $\mu^{1 / 2}$ ) of sc metals for $T<T_{\mathrm{C}} / 2$ is given by $R_{\mathrm{BCS}}=$ $\left.A \cdot f^{\mathrm{n}} \cdot \exp \left(-\Delta^{\prime} / T\right)\right) / T$, with the temperature $T$ measured in $\mathrm{K}$, the frequency $f$ in $\mathrm{GHz}$, and the constants from Table 2 . There is a large potential of attaining a low surface resistance in these compounds, provided that $R_{\text {res }}$ can be made sufficiently low (Fig. 4 ).
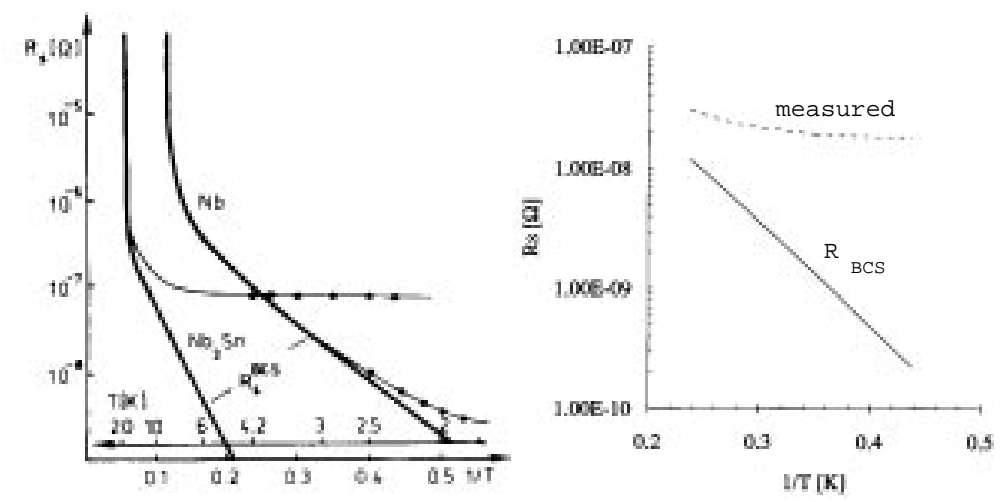

Figure 4. The surface resistance at $500 \mathrm{MHz}$ of a re-entrant cavity with niobium and $\mathrm{Nb}_{3} \mathrm{Sn}$ surface $(\text { left })^{39}$, and that of a NbTiN layer on a copper accelerating mono-cell cavity (right) ${ }^{40}$.

Thin coatings of these materials on RF cavities are produced by thermal diffusion out of the vapour phase at a temperature of 930-1160_C and 1400_C for $\mathrm{Nb}_{3} \mathrm{Sn}^{39,41,42,43,44}$ and $\mathrm{NbN}^{45}$, respectively. Recently, CEBAF has obtained in a $\mathrm{Nb}_{3} \mathrm{Sn}$ coated single-cell 1.5 $\mathrm{GHz}$ cavity (in collaboration with Wuppertal) a Q-value at $4.2 \mathrm{~K}$, which exceeds the design Q-value for $\mathrm{Nb}$ at $2 \mathrm{~K}^{46}$ at the design field of $5 \mathrm{MV} / \mathrm{m}$.

\section{Thin Superconducting Films on a Separate Structure}

In an sc metal the London penetration depth $\lambda$ of the RF field is independent of the frequency in a wide range up to tens of $\mathrm{GHz}, 39 \mathrm{~nm}(\mathrm{Nb})$ and $37 \mathrm{~nm}(\mathrm{~Pb})$. Therefore, to keep the RF losses low, an sc layer of say ten times the penetration depth is largely sufficient. Such films can be produced by several methods, as follows.

Coating by electroplating. Lead can be deposited on a copper substrate by electrodeposition. Although niobium is superior to lead (larger $T_{\mathrm{c}}, B_{\mathrm{c}}$ and lower BCS surface resistance, Table 2), nevertheless lead is particularly used for heavy ion structures 37 in small series at low frequencies (where BCS losses are low), if the geometry is such that high thermal gradients may occur, or when the full potential of RF superconductivity technology is not of paramount importance. Several pre-plating, plating and post-plating treatments have been developed, including plating with $\mathrm{Pb}-\mathrm{Sn}^{38}$ and $\mathrm{Pb}-$ $\mathrm{Bi}^{47}$ alloys.

Coating by sputtering. At $\mathrm{CERN}^{48}$, coating a cavity made of OFE copper by a niobium film has been chosen for several reasons. First, a thermal breakdown (quench) is improbable. Secondly, the material costs would have amounted to about $22 \%$ of the total cavity and cryostat production costs if made from niobium sheet. They are negligible if made from copper sheet. Sputtering has been chosen because the sputtered atoms have a large kinetic energy, which favours the adhesion. DC Magnetron sputtering has been preferred to diode sputtering because of relatively large deposition rates, easy application to multi-cell cavities, and good thickness uniformity. A Q vs. $E_{\text {a }}$ curve is shown in Fig. 3 (b). Compounds as NbN and NbTiN have also been investigated [Fig. 4 (b) $]^{48,49}$.

In the final phase of the industrial series production for CERN, the acceptance rates in initial vertical tests is $60-70 \%\left(E_{\mathrm{a}}=6 \mathrm{MV} / \mathrm{m} \text { and } Q=4 \cdot 10^{9}\right)^{32}$. 
CONCLUSION

Cost-effective mass production is a key issue for accelerating structures for linear colliders. A strong development effort is needed for the following:

- fabrication methods which allow a substantial reduction in production costs (such as, for example, hydroforming, spinning, galvanoplating) and

- more stringent quality control in a fully automated production chain.

\section{REFERENCES}

1. W. Weingarten, Superconducting cavities, Int. J. Mod. Phys. A (Proc. Suppl.) 2B:678 (1993), or Proc. XVth Int. Conf. High Energy Acc., J. Rossbach, ed., World Scientific, Singapore (1993), p. 678.

2. W. Bauer, Fabrication of niobium cavities, Proc. 1st Workshop RF Supercond., M. Kuntze, ed., Karlsruhe (1980), KfK 3019, p. 271.

3. M. G. Rao and P. Kneisel, Thermal and mechanical properties of electron beam welded and heattreated niobium for TESLA, Proc. 6th Workshop RF Supercond., R. Sundelin, ed., CEBAF, Newport News VA, USA (1993), p. 643.

4. A. Combescure, Manuel d'utilisation CASTEM 2000, Rapport CEA DEMT 88/176.

5. J. Genest, private communication.

6. P. H. Ceperley, Ponderomotive oscillations in a superconducting helical resonator, IEEE Trans. Nucl. Sci. NS-19:217 (1972).

7. K. K. Schulze, Preparation and characterization of ultra-high-purity niobium, Journ. of Metals 33:33 (1981).

8. H. Padamsee, The technology of Nb production and purification, Proc. 2nd Workshop RF Supercond., H. Lengeler, ed., CERN Geneva (1984), p. 339.

9. M. Hörmann, The production of high thermal conductivity niobium on a technical scale for high frequency superconductors, available from W.C. Heraeus GmbH, Produktbereich Sondermetalle, P.O. Box 1553, D-6450 Hanau 1, Germany.

10. E. Drost and M. Hörmann, Mass Production of Superconducting Materials $\mathrm{Nb}$ and NbTi, available from W.C. Heraeus GmbH, Produktbereich Sondermetalle, P.O. Box 1553, D-6450 Hanau 1, Germany.

11. P. Kneisel, Improvements in materials and fabrication methods - large scale production and testing Proc. 5th Workshop RF Supercond., DESY 1991, D. Proch, ed., Hamburg (1992), DESY Report M92-01, p. 163.

12. H. Padamsee, A new purification technique for improving the thermal conductivity of superconducting Nb microwave cavities,IEEE Trans. Magn. MAG-21:1007 (1985).

13. P. Kneisel, Use of titanium solid state gettering process for the improvement of the performance of superconducting RF cavities, J. Less Common Metals 139:179 (1988).

14. Columbium (Niobium) Alloys and special products from Teledyne Wah Chang Albany, OR, USA.

15. Columbium (Niobium) from Kawecki Berylco Industries, Inc., Reading PA, USA.

16. Niobium / Columbium from Companhia Brasileira de Metalurgia e Mineração, São Paolo, Brazil.

17. J. P. Bacher et al., Brazing of niobium to stainless steel for UHV application in superconducting cavities, Internal Report CERN/EF-RF 87-7, Dec. 1987.

18. J. Susta, Development of Fabrication Methods, ibid. ref. 8, p. 597.

19. J. Kirchgessner, Forming and Welding of niobium for superconducting cavities, Proc. 3rd Workshop RF Supercond., K. W. Shepard, ed., Argonne Nat. Lab. (1988), ANL-PHY-88-1, p. 533.

20. T. Furuya, Preparation and handling of superconducting RF cavities, Proc. 4th Workshop RF Supercond. KEK, Tsukuba, Japan, 1989, KEK Report 89-21 (January 1990), p. 305.

21. D. Bloess et al., Superconducting, hydroformed, niobium sputter coated copper cavities at $1.5 \mathrm{GHz}$, Proc. 4th European Particle Acc. Conf. London 1994, V. Suller, Ch. Petit-Jean-Genaz, eds., Singapore (1994), p. 2057.

22. C. Hauviller, Fully hydroformed RF cavities, Proc. Particle Acc. Conf. Chicago 1989 (IEEE, New York, 1989), p. 213.

23. E. Chiaveri and H. Lengeler, Welding of Niobium Cavities at CERN, ibid. ref. 8, p. 611.

24. E. Chiaveri et al., Welding of radio frequency cavities with an internal EB gun, Proc. First Int. Conf. Power Beam Technology, Brighton, UK, 1986, The Welding Institute, London.

25. A. Citron et al., The Karlsruhe-CERN superconducting RF separator,Nucl. Instr. Meth. 164:31 (1979).

26. Ph. Bernard et al., Superconducting hydroformed niobium sputter coated copper cavities at $1.5 \mathrm{GHz}$, ibid. ref. 3, p. 739.

27. D. Bushnell, BOSOR5 Program for buckling of complex , branched shells of revolution including large deflections, plasticity and creep, Lockheed Applied Mechanics Lab.

28. J. Turneaure et al., Performance of 6-m $1300 \mathrm{MHz}$ superconducting niobium accelerator structures, Appl. Phys. Lett. 25:247 (1974). 
29. V. Palmieri et al., A new method for forming seamless $1.5 \mathrm{GHz}$ multicell cavities starting from planar disks, ibid. ref. 21, p. 2212.

30. A.I. Ageev et al., Development of production technology for weldless copper shells of sc cavities, ibid. ref. 3, p. 802.

31. E. Kako et al., Long term performance of the TRISTAN superconducting RF cavities, Proc. 1991 IEEE Particle Acc. Conf. San Francisco, USA, 1991, (IEEE, New York, 1991), p. 2408.

32. E. Chiaveri et al., Analysis and results of the industrial production of the superconducting $\mathrm{Nb} / \mathrm{Cu}$ cavities for the LEP2 project, Proc. XVIth Int. Conf. High Energy Acc., Dallas TX, USA, 1995, in press.

33. C. Reece et al., Performance of Production SRF Cavities for CEBAF, Proc. 1993 IEEE Part. Acc. Conf. , Piscataway, NJ, USA, (1993), p. 1016.

34. B. Aune, private communication.

35. M. Leenen, The infrastructure for the TESLA Test Facility (TTF) - a status report, ibid. ref. 30, p. 2060.

36. B. Bonin and R. W. Röth, Q-degradation of niobium cavities due to hydrogen contamination, ibid. ref. 11, p. 210.

37. J. R. Delayen, RF properties of superconducting Pb electroplated onto $\mathrm{Cu}$, ibid. ref. 19, p. 469.

38. L. Dietl and U. Trinks, The surface resistance of a superconducting lead-tin alloy, Nucl. Instr. Meth. A284:293 (1989).

39. G. Arnolds-Mayer and N. Hilleret, Field emission and secondary electron emission from $\mathrm{Nb}_{3} \mathrm{Sn}$ surfaces, Advanc. in Cryog. Eng. Mat. 28:611 (1982).

40. C. Benvenuti et al., (NbTi)N and NbTi coatings for superconducting accelerating cavities, ibid. ref. 11, p. 518.

41. G. Arnolds-Mayer, ibid. ref. 8, p. 643.

42. M. Peiniger et al., Nb3 Sn for superconducting accelerators at 4.2 K, Proc. First European Acc. Conf., Rome 1988, S. Tazzari, ed., Singapore (1989), p. 1295.

43. G. Arnolds-Mayer and E. Chiaveri, On a $500 \mathrm{MHz}$ single cell cavity with $\mathrm{Nb}_{3} \mathrm{Sn}$ surface, ibid. ref. 19, p. 491.

44. B. Hillenbrand et al., Superconducting Nb33 cavities IEEE Trans. Magn. MAG-11:420 (1975).

45. G. Gemme et al., RF surface resistance measurements of binary and ternary niobium compounds, J. Appl. Phys. 77:257 (1995).

46. H. Piel, private communication.

47. J. Sikora et al., Lead/Tin Resonator Development at the Stony Brook Heavy-Ion Linac,, ibid. ref. 19, p. 419.

48. C. Benvenuti et al., Niobium films for superconducting accelerating cavities, Appl. Phys. Lett. 45:583 (1984); C. Benvenuti et al., Superconducting $500 \mathrm{MHz}$ accelerating copper cavities sputter-coated with niobium films, IEEE Trans. on Magn. MAG-21:153 (1985).

49. S. Cantacuzène et al., Elaboration et caractérisation de couches minces de $\left(\mathrm{Nb}_{\mathrm{x}} \mathrm{Ti} \mathrm{T}_{1-\mathrm{x}}\right) \mathrm{N}$ pour des applications en hyperfréquences, thesis, Université de Paris Sud, centre d'Orsay (1995). 